\title{
EVALUACIÓN DE LA MODALIDAD DE INTERACCIÓN DE LA TUTORÍA Y LOS EFECTOS EN LOGRO ACADÉMICO EN ENTORNOS EN LÍNEA
}

\author{
(AN EVALUATION OF THE INTERACTIVE MODALITY \\ OF TUTORIALS AND ITS EFFECTS ON ACADEMIC \\ ACHIEVEMENT IN ONLINE LEARNING ENVIRONMENTS )
}

\author{
Omar Moreno Almazán \\ SUAED UNAM Iztacala, México
}

\section{RESUMEN}

Existen muchos elementos que pueden brindarnos información sobre los efectos de los programas de aprendizaje en las distintas modalidades, aun cuando los indicadores más aceptados por las instituciones sean los relacionados con el rendimiento y con la permanencia de la matrícula estudiantil; sin embargo la acción del docente suele quedar relegada o incluso justificada en su legítima naturaleza. El presente trabajo corresponde a una evaluación de los efectos que existen en la interacción y desempeño del tutor sobre el rendimiento de los alumnos en un sistema e-learning. Este efecto se traduce en indicadores de logro académico que han sido estudiados en un grupo de 27 estudiantes de licenciatura en la modalidad de educación a distancia, a través de una exploración basada en análisis descriptivos, así como de la determinación de efectos mediante el análisis de varianza factorial, a fin de no situarse solo en el cumplimiento de índices de aprobación sino haciendo un estudio global de todos los casos en cuanto a su adquisición de conocimientos y habilidades. Entre los resultados destaca una acción docente basada en comunicación pero también en elementos cognitivos que brinden razonamiento, motivación y retroalimentación; sus implicaciones siguen siendo eje de discusión sobre la dinámica de enseñanza en esta modalidad, en virtud de que algunas instituciones mantienen la idea de atribuir mayor carga formativa a los estudiantes con la intención de lograr un mejor aprendizaje.

Palabras clave: educación a distancia, métodos de enseñanza, logro académico, análisis de los procesos de interacción.

\begin{abstract}
There are many elements that can give us information on the effects of learning programs in different modes, even when indicators accepted by more institutions are related to performance and retention of student enrollment; but the action of the teacher usually be relegated or even justified in their legitimate nature. This paper is an assessment of the effects that exist in interaction and performance tutor on student performance in an e-learning
\end{abstract}


system. This effect results in academic achievement indicators that have been studied in a group of 27 undergraduate students in the distance education mode, through an exploration based on descriptive analysis and determination of effects by analyzing factorial variance in order not to stand alone in meeting approval ratings but making a comprehensive study of all cases in their acquisition of knowledge and skills. It finds a teaching action based on communication but also in cognitive elements that provide reasoning, motivation and feedback; their implications remain shaft discussion on the dynamics of teaching in this mode, under which some institutions maintain the idea of giving more load training to students with the intention of achieving better learning.

Keywords: distance education, teaching methods, academic achievement, interactions process analysis.

Cada vez son más los estudios que se enfocan en comprender la relación que se está dando entre docentes y alumnos en un entorno virtual de aprendizaje, siendo que al principio se daba una continua comparación entre la enseñanza en entornos educativos tradicionales con carácter presencial y los entornos en línea y virtuales; muchos de los cuales apoyaron la idea de que los entornos presenciales superan los resultados en comparación con los entornos virtuales (Carroll, 2004; Edmonds, 2006; Redding y Rotzien, 2001; O’ Malley, 1999); sin embargo, es necesario destacar que tras la aparición de entornos educativos virtuales y su alta demanda en la sociedad, se ha generado la presencia de modelos de interacción que crean efectos en la relación y dinámica educativa.

A decir de Anderson y Kuskis (2007) existen seis tipos de interacción que han investigado en las formas de cómo se desarrolla la educación a distancia y que involucran principalmente la relación que se da entre herramientas, elementos pedagógicos y tecnológicos. Las interacciones que se citan son:

- Interacción entre alumno-tutor y alumno-institución. Consiste principalmente en la relación que se genera a partir de la forma en la que se involucra el tutor docente, los alumnos y el contenido dentro de un ambiente de aprendizaje.

- Interacción entre alumno y alumno. Explica la forma en la que los alumnos, incluidos dentro de un ambiente de aprendizaje, generan relación académica y de estrategia a nivel individual y colaborativo. Implica el acompañamiento que entre miembros se dan y aumenta una mejor condición y motivación, intercambio de ideas y redes de comunicación.

- Interacción entre alumno y contenido. El modo en que se presenta el contenido mediante distintos medios es lo que provoca diferentes formas de relación y asimilación de los alumnos con las temáticas de la educación a distancia. Se estudian las formas que se suelen emplear (archivos de sonido, de texto, gráficos, video y realidad virtual) y el impacto que cada una de estas genera. El estudio de 
esta relación implica el análisis a nivel del uso de tecnologías didácticas y de la formas de estructuración cognitiva que se generan. Vale decir que esta es una de las formas de interacción que más se estudian y han generado impacto.

- Interacción entre tutor y contenido. De manera contraria esta es una de las formas menos estudiadas en el ámbito de la educación a distancia; tiene una relación directa con el estudio de los procesos de diseño instruccional y los desarrollos creados por los tutores, a fin de dosificar la información y asignar actividades.

- Interacción entre tutor y tutor. A decir de los autores, el uso de recursos de enseñanza en línea proporciona oportunidades infinitas de interacción entre docentes, que pueden incrementar y compartir sus habilidades pedagógicas y propiciar mayores alcances de intercambio de información en las formas de impartir docencia. Es una relación también poco explorada a nivel educativo, pero sí llena de información en cuanto a los recursos materiales e incluso comerciales que se brindan para su divulgación.

- Interacción entre contenido y contenido. Es un estudio que comienza a revisarse y es de interés de científicos computacionales y creadores educativos. Está basado en la relación entre programas y sistemas "inteligentes" que pueden generar diferentes formas a las convencionales de programas para la educación y que sean de larga duración, sistemas semiautónomos, proactivos y adaptativos.

El interés de este estudio se centra en conocer la relación entre alumno y tutor y su impacto en la generación de habilidades de aprendizaje autorregulado. Es preciso indicar que el principal beneficio pedagógico de esta relación comienza con la motivación y la retroalimentación; la cual puede darse por medios sincrónicos y asincrónicos, habiendo estudios que demuestran su efectividad (Wang, 2004; Katz, 2000); pero además, otro de los elementos presentes en dichos estudios es el costo de la interacción humana, la cual tiende a incrementar proporcionalmente con relación al número de estudiantes.

La principal connotación conlleva a entender que no se concibe a un tutor de un sistema de educación a distancia señalando que únicamente responderá correos electrónicos en un horario específico, puesto que la interacción entre alumnos y tutores está basada en forma que se perciba su presencia social y la inmediatez que los alumnos perciban de las retroalimentaciones e información de su tutor; entendiendo como presencial social el grado en que se percibe a una persona en forma "real" en cuanto a su intencionalidad y la presencia de su enseñanza (Mc.Isaac y Gunawardena, 1996). Esta presencia social se traduce en un sentido docente, en el diseño, facilidad y dirección de procesos cognitivos y sociales con el propósito de lograr resultados de aprendizaje personalmente significativos e importantes (Anderson, Rourke, Garrison 
y Archer, 2001). El efecto que resulta de la presencia de enseñanza significativa debe reflejarse en una lógica en la que, a mayor efecto positivo, la percepción y procesos cognitivos se amplían en comparación con estudiantes que tienen menor inmediatez en la comunicación de sus tutores (Backer, 2004).

Una de las propuestas de interacción explicativa entre alumnos y tutores en un sistema a distancia, se puede ver en el modelo instruccional propuesto por Garrison y Anderson (2003) en la que se propone un sistema formal de educación a distancia, en donde la interacción es principalmente mediada por el tutor, pero que dicha interacción se extiende hacia un impacto generalizado de la web y que se refleja en los servicios a los estudiantes y en la automatización que se dirija a una interacción entre el estudiante y la institución (figura 1).

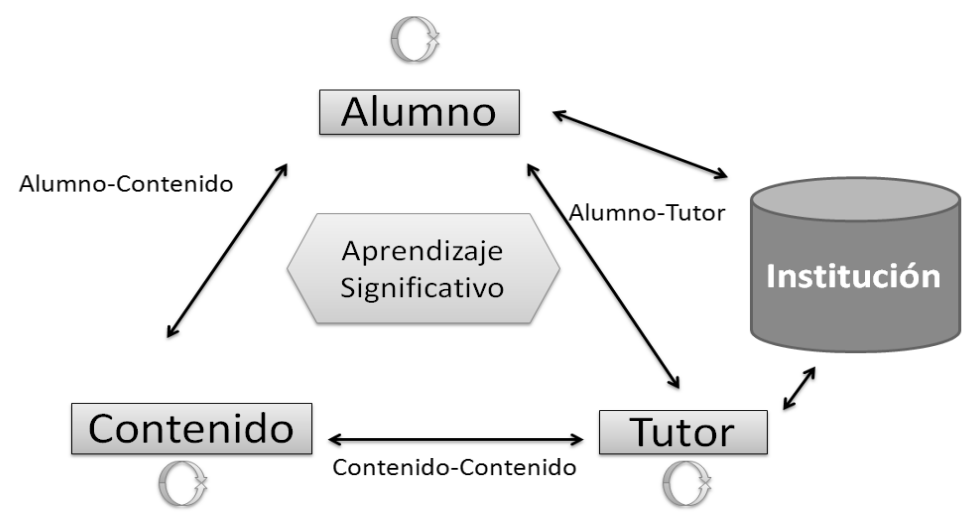

Figura 1. Modos de interacción en un sistema de educación a distancia, desde el modelo instruccional de Garrison y Anderson (1998)

Como se puede apreciar, en la propuesta del modelo de interacción de Garrison y Anderson (op. cit.), es el tutor quien mantiene el punto de interacción principal, el cual genera una relación con el contenido a partir de los diseños y objetos que utiliza en los distintos portales, sitios o plataformas en la red y, por otra parte, con las vistas personalizadas de la institución a su labor, aunado a los servicios para los alumnos y las expectativas que se tienen de ellos.

En este sentido y de modo directo, el proceso de interacción que se genera en un sistema de educación a distancia involucra la participación de contenido, herramientas, institución, servicios estudiantiles y tutores con el fin de promover un proceso de aprendizaje significativo, en donde el alumno es el destinatario de todas estas interacciones. 
De manera particular en relación al papel que debiera asumir un tutor en educación a distancia, implica la necesidad de propiciar una guía en el proceso de aprendizaje. Su rol debiera incluir competencias para la organización de los temas, la preparación de métodos instruccionales y para la enseñanza a distancia como tal (Simonson, Smaldino, Albright y Zvacek, 2003).

En el sentido de la relación con factores pedagógicos, las habilidades para preparar métodos instruccionales constituyen un elemento que en principio debiera estar basado en las características de la forma de enseñanza del tutor, en sus estudiantes, contenidos y los sistemas de entrega; debido a que la mayor responsabilidad de aprendizaje recae sobre los alumnos y que por ende los métodos deben focalizarse en incluir procesos interactivos que permitan mayor éxito a los alumnos. Además, se debe considerar el uso de técnicas que puedan reducir los sentimientos que algunos alumnos experimentan gradualmente, donde se pueda involucrar a todos los alumnos desde el momento de la presentación y que hagan del entorno virtual más confortable, propiciando la participación espontánea de los mismos alumnos (Simonson, Smaldino, Albright y Zvacek, 2003).

En cuanto al papel del tutor docente sobre las habilidades de autorregulación, se debe señalar que genera condiciones que influyen en las habilidades de los alumnos. En un modelo presentado por Azevedo (2005) se realiza un protocolo que analiza los esquemas de aprendizaje autorregulado de los alumnos y en el cual destaca cinco variables que son extraídas al momento de analizar el ejercicio de trabajo de los alumnos en entornos de hipermedia. En dicho esquema se consideran actividades determinadas por el tutor:

- Planeación. (Actividades de planeación de objetivos, activación de conocimiento previo y rehúso de metas en la memoria de trabajo);

- Monitoreo. (Opinión de lo que se aprende, sentimientos de dominio de conocimiento, autocuestionamientos, evaluación de contenido e identificación de la información adecuada en los entornos virtuales);

- Uso de estrategias. (Formas para seleccionar nueva información, investigación, resumen, copia de la información, repetición de la lectura, uso de inferencias, hipotetizar, elaboración de conocimiento y evaluación de contenidos como respuesta a preguntas hechas);

- Demanda y dificultad de tareas. (Planeación de tiempo y esfuerzo, conductas de ayuda, tareas difíciles, control de contexto de aprendizaje y expectativas de adquisición de aprendizaje); y, 
- Declaración de interés. (Nivel de interés del alumno en tarea/tema/dominio) (Azevedo, 2005).

Cada una de estas actividades presenta una relación directa con las formas en las que el alumno comienza a desarrollar habilidades autorregulatorias que generan éxito en las actividades de aprendizaje.

De esta forma es sabido que entre las interacciones que se dan en los sistemas de educación a distancia, existen relaciones directas en donde el alumno es la pieza central y el objetivo principal, mientras que el docente tutor es la figura primaria que desempeña una relación con todos los elementos educativos. La función tutora generará impacto en la interrelación a partir de crear algunas habilidades autorregulatorias que, a decir de Vohs y Ciarocco (2004), se traducen en:

- Orientación social. Donde se considera que los responsables del proceso son los responsables de crear formas de control mediante las reglas y que en consecuencia pueden traer mejores resultados y formas de autorregularse. El papel se centra en fungir como orientador en el desarrollo de tareas y la participación gradual de los involucrados.

- Autopresentación. La autopresentación y la autorregulación están relacionadas en el sentido de que la primera procura la generación de impresiones sociales positivas a partir de los logros obtenidos y, es quizá, uno de los elementos que tiene alta influencia en el actuar de los individuos; pero además, requiere de la dirección de respuestas que pudieran afectar al desarrollo de la formación tales como acciones impulsivas, emocionales y respuestas cognitivas.

- Resistencia como forma de autocontrol. En este sentido se manejan dos elementos: por un lado los intentos por aumentar las fuerzas ante ciertas circunstancias dentro del desarrollo formativo (intentos alfa) y la reducción de fuerza de oposición (intento omega). La acción tutora se encauza en procurar por el equilibrio entre estos elementos.

Con base a la identificación de factores queinvolucran la interacción entrela acción docente y las habilidades de autorregulación en el proceso formativo de alumnos en sistemas de educación a distancia, es posible deducir que en un sistema basado en el e-learning en la carrera de psicología se pueden generar logros académicos a partir del fomento de habilidades de autorregulación en los estudiantes. Se parte de la idea de que el desarrollo de estas habilidades se traduce en éxito académico y que este tiene relación con las habilidades que el tutor genera a partir de su interacción. 


\section{MÉTODO}

Objetivo: identificar y evaluar los efectos de la interacción que establece un tutor a través de medios de educación virtual, a partir de los indicadores del uso de recursos y acciones del tutor, de sus interacciones y de los efectos en el rendimiento de los alumnos.

Justificación: Los modelos de enseñanza basados en enfoques cognoscitivos, parten de la noción de que los alumnos se conviertan en expertos a partir de procesos de andamiaje; estos procesos deben estar basados en una comunicación continua y en interactividad. Para el cumplimiento de esta condición, se exige que el tutor adquiera primero dominio en estos procesos así como el conocimiento en el uso de herramientas que faciliten el desarrollo de tales procesos.

Tipo de investigación: Descriptiva y correlacional.

Diseño de investigación: Transeccional, no experimental.

Participantes: El estudio se desarrolló con 27 estudiantes de la Facultad de Estudios Superiores Iztacala, inscritos en la asignatura de Psicología Teórica I: Historia y Antecedentes de la Psicología Clínica, del Sistema de Universidad Abierta y Educación a Distancia (SUAED). Ninguno de los estudiantes había estado anteriormente bajo una programación diseñada en modelos de autorregulación del aprendizaje y, era además la primera vez que cursaban un módulo de profundización en el área de la psicología clínica, teniendo escasos conocimientos al respecto de esta materia.

Materiales: El desarrollo del módulo se basó rigurosamente en el contenido autorizado en el Plan de Estudios del SUAED, Psicología; y, el desarrollo de actividades se encauzaron en los contenidos específicos, así como el empleo de lecturas seleccionadas y digitalizadas que dieran cobertura a las unidades mínimas de aprendizaje.

El diseño instruccional del módulo está basado en los principios de Merill (2002) y se compone de: a) Planteamiento inicial de un problema para conducción y activación instruccional; b) Activación del conocimiento disponible; c) Demostración del conocimiento; d) Facilitación de la integración del conocimiento basado en la aplicación de conocimiento a nivel práctico y en la solución de problemas; así como en el de Anderson \& Garrison (1998) donde se especifica el modelo de interacción entre los agentes educativos. 


\section{Recurso Digital}

El sistema en línea que se utilizó para este estudio es una plataforma virtual de aprendizaje basada en Moodle denominada Consorcio Universitario Virtual de Educación a Distancia (CUVED) y en el cual los alumnos se incluyen e ingresan a sus sesiones de trabajo mediante el acceso de un usuario y contraseña. Cuando ingresan los usuarios pueden visualizar la estructura del programa, actividades y recursos virtuales.

El CUVED dispone adicionalmente de algunos elementos que permiten generar registros sobre el desempeño de sus usuarios y sobre los procesos de interacción entre los diferentes agentes educativos que permite generar estimaciones de la actividad en estas interacciones. Entre estos elementos destaca:

- Registros de visitas y desempeño. Un área en la que es posible llevar el registro del número de accesos tanto a la plataforma como a cada uno de los recursos incluidos; este registro permite llevar también una secuencia cronológica del número de visitas de cada usuario.

- Registro de cantidad de retroalimentación. En donde se disponen de registros numéricos del número de retroalimentaciones que hace el tutor sobre las actividades de los alumnos, así como la cantidad de elementos incluidos en cada retroalimentación.

- Registro de latencia de retroalimentación. En el cual se registra el tiempo que transcurre desde que cada usuario realiza o sube una actividad y es retroalimentada y evaluada por el tutor.

- Sistema de comunicación. El cual se basa en el desarrollo de mensajería y se construye por formas de comunicación a nivel de grupo, subgrupos o por usuario.

Entre las actividades que se incluyeron en el CUVED y que están basadas en los modelos de autorregulación, se incluye una bitácora que expone las metas de aprendizaje y los tiempos de ejecución, la elaboración de notas para cada trabajo realizado, un cronograma de actividades, consulta libre de recursos virtuales y la escala de monitoreo de desempeño.

La estructura de aprendizaje del CUVED está construida por tres componentes verticales principales. En el área izquierda se incluían las actividades de aprendizaje y monitoreo, construida por vínculos que conducen directamente a cada actividad y a los registros de desempeño y calificación de cada alumno. El área derecha se compuso de elementos principalmente informativos en dos niveles: sobre aspectos relacionados con la conducción instruccional del módulo y sobre eventos académicos 
extracurriculares que ampliaban el panorama formativo basado en el conocimiento del módulo. El área central disponía de pestañas, destinadas cada una a las Unidades Mínimas de Aprendizaje y una pestaña principal de Presentación del Módulo; en estas pestañas se tenía la información sobre los procedimientos de aprobación del módulo, los recursos específicos, documentos electrónicos, actividades y medios de comunicación. El acceso a todas las áreas fue abierto durante todo el tiempo que duró el semestre y jamás reportó alguna caída de sistema o problemas de conectividad.

De manera adicional se dispuso del sistema de comunicación visual de acceso gratuito USTREAM que permite la realización de sesiones tipo videoconferencias con los recursos mínimos, ya que solo se requiere de una computadora conectada a la red, una webcam y dispositivos de salida de audio. Este sistema tiene entre sus bondades, que dirige su comunicación de manera libre o restringida a ciertos usuarios, se puede grabar la sesión y se conecta a las principales redes de comunicación como son los chats de Facebook o Twitter, además de contar con su propio sistema de comunicación sincrónica. El alumno puede acceder a las sesiones en el momento en vivo o visitarlas cuantas veces lo desee en momentos posteriores, es decir está disponible en todo momento.

\section{Procedimiento}

Se tomó a un grupo de un módulo de quinto semestre de la carrera de psicología en el sistema de educación a distancia, quienes dentro de su plan de estudios han acreditado un tronco común formativo y se estima en ellos una adaptación relativa al sistema.

Los integrantes de este grupo fueron incluidos en el programa basado en modelos de aprendizaje autorregulado con sus respectivas condiciones instruccionales. La secuencia de trabajo instruccional basada en el programa de autorregulación fue la siguiente:

Tabla 1.

Estructura básica del programa de autorregulación en aula virtual

\begin{tabular}{|c|c|c|c|c|c|c|c|c|c|}
\hline $\begin{array}{l}\text { Fase de } \\
\text { presen- } \\
\text { tación e } \\
\text { inicio }\end{array}$ & $\begin{array}{l}\text { Estableci- } \\
\text { miento de } \\
\text { metas }\end{array}$ & $\begin{array}{l}\text { Actividad } \\
\text { inductiva }\end{array}$ & UMA 1 & Ev 1 & UMA 2 & Ev 2 & UMA 3 & Ev. 3 & $\begin{array}{l}\text { Evalu- } \\
\text { ación } \\
\text { final }\end{array}$ \\
\hline $\begin{array}{l}\text { Perfil y } \\
\text { presen- } \\
\text { tación }\end{array}$ & $\begin{array}{l}\text { Crono- } \\
\text { grama y } \\
\text { agenda }\end{array}$ & $\begin{array}{l}\text { Acti- } \\
\text { vidad } \\
\text { colabo- } \\
\text { rativa }\end{array}$ & & $\begin{array}{l}\text { Acti- } \\
\text { vidad } \\
\text { colabo- } \\
\text { rativa }\end{array}$ & & $\begin{array}{l}\text { Acti- } \\
\text { vidad } \\
\text { colabo- } \\
\text { rativa }\end{array}$ & & $\begin{array}{l}\text { Acti- } \\
\text { vidad } \\
\text { colabo- } \\
\text { rativa }\end{array}$ & $\begin{array}{l}\text { Autoe- } \\
\text { valua- } \\
\text { ción e } \\
\text { inte- } \\
\text { gración } \\
\text { final }\end{array}$ \\
\hline
\end{tabular}


Una vez inscritos los alumnos al módulo y matriculados en el CUVED, ellos disponían de un tiempo común sin control sincrónico de entrada, ya que disponían de las 24 horas de cada día que duró el semestre para que entraran en el momento que considerasen pertinente. Se disponía de plazos para el desarrollo de cada actividad y estos plazos eran el referente para que cada alumno estableciera sus propios plazos personales dentro de un cronograma que ellos mismos hacían, mismos que fueron monitoreados continuamente. Vale decir que los alumnos que participaron en este estudio no presentaron en ningún momento una sesión presencial obligada; lo más cercano a ello fue el desarrollo de sesiones visuales en línea sincrónica y asincrónica de manera voluntaria (figura 2). Así mismo hubo la presencia de alumnos de diferentes estados de la república, siendo principalmente de Durango, Oaxaca, Puebla, Hidalgo, Estado de México, Tlaxcala, Jalisco, Querétaro y Distrito Federal, así como una alumna que radica en San Diego, Estados Unidos de América.

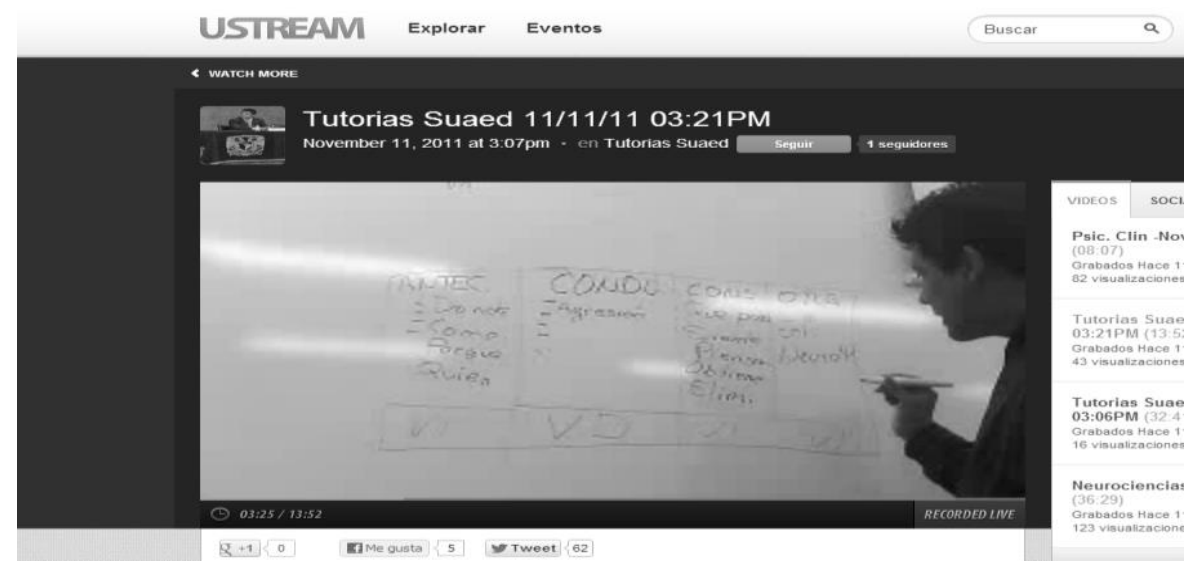

Figura 2. Ejemplo de una sesión sincrónica en línea a través del programa USTREAM

Al incluir a los estudiantes a la plataforma CUVED en la cual se destinó un periodo de conocimiento del entorno y del ajuste de sus perfiles, programación autodirigida y del contenido temático; se procuró porque todos los estudiantes contarán con estas acciones en un periodo máximo de 15 días después de haber iniciado el semestre. Las actividades formativas se les brindaron mediante una programación de la cual debieron realizar un cronograma con base a un calendario semestral, mismo que se adjuntó a la plataforma como referente de avance de cada alumno. Se les indicó que el entorno había sido diseñado para promover un aprendizaje autorregulado y que contarían con el apoyo continuo del tutor, pero que la determinación de objetivos por unidad y de metas académicas estaba sujeta al resultado de cada uno mediante el seguimiento instruccional otorgado en la programación. Se les señaló 
que la reciprocidad en la comunicación y formación estarían sujetas principalmente a la retroalimentación de cada tarea y en la participación tanto sincrónica como asincrónica; los medios para ello estarían establecidos en la plataforma CUVED.

\section{Variables}

Para conocer los indicadores relacionados con la labor docente sobre los alumnos, se consideraron los resultados obtenidos de las frecuencias en el uso de actividades de interacción y comunicación. Estos indicadores fueron activados con el fin de conocer la frecuencia y magnitud de estas variables y su impacto sobre el desempeño docente. No se utilizaron instrumentos específicos, sino que los indicadores que se generaron desde la plataforma CUVED.

Los indicadores se situaron en diferentes niveles los cuales eran determinados por CUVED:

Se empleó el registro de actividades en cada visita dada por el tutor; este registro presenta la frecuencia de acceso y las visitas hechas en cada sitio, así como cada movimiento interno dentro del CUVED. En este mismo registro se obtiene la cantidad de retroalimentaciones que son dadas a cada tarea y a cada alumno.

Otro de los indicadores obtenidos es el tiempo que se genera entre la actividad hecha por cada alumno y la respuesta del tutor; lo que propiamente se considera la latencia de respuesta que hay en cada retroalimentación dada a partir de que el alumno expone una duda o adjunta una actividad.

Del mismo modo se obtiene el número de ocasiones en las que se emplea comunicación directa e indirecta; esto es el número de veces en las que se usó la mensajería individual y grupal, las retroalimentaciones, las acciones colaborativas y los mecanismos de retroalimentación visual; todo ello generando sus registros (figura 3). 


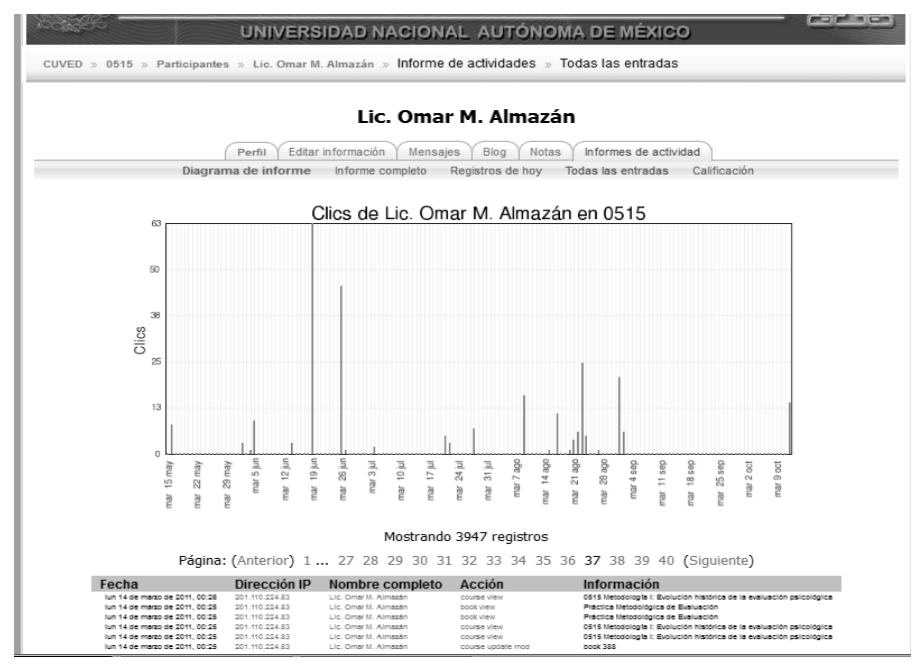

Figura 3. Gráfica de registro de actividad de un usuario en plataforma Moodle

Se contó con los registros señalados y que se iban acumulando en tiempo real tras la actividad de cada alumno. El tipo de escala de estos registros obedece a que es la forma en la que el CUVED proporcionó registros de las tareas de autorregulación y de las actividades de evaluación, así como de la dinámica de trabajo y de comunicación de los usuarios y del tutor.

\section{RESULTADOS}

Los resultados de este estudio se dividen en tres partes principales: a) El análisis de las evaluaciones obtenidas y los recursos empleados, b) Descripción de la actividad tutorial basada en programas instruccionales de modelos autorreguladores, y c) Efectos de la acción tutorial sobre el desempeño y habilidades de los alumnos.

- Análisis de las evaluaciones obtenidas y los recursos empleados

Mediante un estudio de análisis de varianza que se aplicó a los resultados obtenidos por los alumnos en cada una de las actividades del programa a fin de determinar efectos significativo que hubo a nivel de intra actividades $F(4)=.366, p$ $>0.05$, demostrando que no hubo actividades que tuvieran un efecto diferente con respecto a las demás, habiendo entre ellas índices de homogeneidad con el mismo valor en cuanto a su efecto sobre los resultados de los alumnos. Adicionalmente se aplicó la prueba de comparación múltiple de Sheffé a fin de estimar algún nivel o 
tendencia significativa entre cada actividad específica, no hallando diferencias en ninguna de las trayectorias o relaciones específicas.

Del mismo modo se realizó un análisis a fin de encontrar las direcciones de cada actividad en su relación directa con la calificación de los alumnos, a través de una prueba de análisis de varianza en la que se encontraron efectos significativos. Para el caso de las actividades colaborativas, el resultado muestra una diferencia significativa $\mathrm{F}(5)=6.224, \mathrm{p}<0.05$; la participación individual presentó un efecto significativo de F (5) = 14.899, p < 0.05; en el caso de las evidencias de aprendizaje, tuvieron un efecto significativo de $\mathrm{F}(5)=14.594$, p < 0.05 y el caso de la integración final presentó un efecto significativo de F (5) = 47.160, p < 0.05.

Se aplican los procedimientos de análisis descriptivo para saber cuál ha sido la tendencia que describe el resultado en los alumnos en términos de su aprendizaje. La tabla 2 muestra la estadística descriptiva de los elementos que compusieron la estructura del programa con respecto a las calificaciones de los alumnos:

Tabla 2.

Estadísticos descriptivos del análisis de actividad en el curso virtual

\begin{tabular}{|l|c|c|c|c|c|c|}
\hline \multicolumn{1}{|c|}{ Actividad } & $\mathrm{N}$ & Media & $\begin{array}{c}\text { Desviación } \\
\text { estandar }\end{array}$ & $\begin{array}{c}\text { Error } \\
\text { estandar }\end{array}$ & Mínimo & Máximo \\
\hline Colaborativas & 27 & 6.96 & 4.62 & .889 & 0 & 10 \\
\hline Participación individual & 27 & 7.33 & 3.669 & .706 & 0 & 10 \\
\hline Evidencias de Aprendizaje & 27 & 6.39 & 3.352 & .645 & 0 & 10 \\
\hline Integración Final & 27 & 7.15 & 3.687 & .710 & 0 & 10 \\
\hline Calificación Final & 27 & 7.52 & 3.203 & .616 & 0 & 10 \\
\hline
\end{tabular}

Es posible observar que tanto a nivel estadístico como a nivel visual, no hay una distinción sobre el peso que genera alguna actividad en específico sobre la calificación; de tal forma que las actividades programadas tuvieron un efecto semejante sobre la calificaciones de los alumnos sin marcar una tendenciosidad respecto a alguna; y sin embargo, dichas actividades si tienen una relación significativa importante sobre el logro en cada alumno.

El resultado final de los alumnos en este módulo se describe en la tabla 3: 
Tabla 3.

Calificaciones finales en el curso virtual

\begin{tabular}{|c|c|c|c|c|c|c|c|c|c|c|c|c|c|}
\hline $\begin{array}{c}\text { NOMBRE } \\
\text { DE LA } \\
\text { ASIGNATURA }\end{array}$ & 10 & 9 & 8 & 7 & 6 & ACRED & 5 & NP & REPROB & INSC & APROBACIÓN & REPROBACIÓN \\
\hline $\begin{array}{c}\text { PSIC.TEOR.I } \\
\begin{array}{c}\text { HIST.Y } \\
\text { ANTECED. } \\
\text { PSICOL.CL }\end{array}\end{array}$ & 10 & 4 & 5 & 1 & 0 & 20 & 4 & 3 & 7 & 27 & $74.07 \%$ & $25.92 \%$ \\
\hline
\end{tabular}

El resultado de la aplicación de un programa basado en mecanismos de autorregulación parece favorecer el nivel de aprobación del módulo $744.07 \%$ de aprobación) y que es contrastante respecto a los índices de no acreditados y más aún de la deserción (de 27 inscritos, desertaron 3 alumnos). En este sentido parece indicarse que el uso de programas que se basen en sistemas de autorregulación, favorece el trabajo de los alumnos y sus logros se ven representados por la acreditación del módulo, pero además con el cumplimiento de casi la totalidad de los elementos evaluativos (50\% de ellos cubren de manera total todas las actividades, sobre 20 acreditados). En términos de calificaciones bajas, el programa otorga pocos alumnos donde apenas si hay uno que obtiene 7 (70\% de cumplimiento en lo que el programa exigió) y ninguno con calificación de seis. Reprueban siete personas las cuales representan a alumnos que si efectuaron al menos una actividad pero no alcanzaron a cubrir las actividades mínimas para acreditar el módulo.

El resultado del grupo se puede describir con una calificación promedio de 7.52, con una desviación estándar de 3.203; donde la curtosis (1.292) indica que la mayor parte de los datos se desplazan del lado positivo de la media.

Descripción de la actividad tutorial basada en programas instruccionales de modelos autorreguladores.

Como elemento importante de este análisis, se consideró útil evaluar el efecto que tuvo la acción docente en el cumplimiento de este programa autorregulatorio. Se hace un análisis de las actividades docentes que tuvieron efecto sobre este programa.

Uno de los primeros componentes fue el considerar la frecuencia de visitas específicas que se hicieron a las tareas y actividades de cada alumno en específico (omitiendo los accesos con intención de trabajo grupal); en el mismo tenor, se hace un recuento de la frecuencia en el número de retroalimentaciones que se hizo a cada alumno, considerando que el programa incluyó: 
- 1 actividad inductiva

- 3 actividades colaborativas

- 3 evidencias de aprendizaje

- 1 integración final

De este modo se tiene el siguiente indicador:

Tabla 4.

Indicadores de acceso al aula virtual

\begin{tabular}{|l|c|c|} 
& $\begin{array}{c}\text { Número de visitas por } \\
\text { tarea/individuo }\end{array}$ & $\begin{array}{c}\text { Número de } \\
\text { retroalimentaciones } \\
\text { por tarea/individuo }\end{array}$ \\
\hline Media & 4.70 & 3.07 \\
\hline Desv. típ. & 1.235 & 1.072 \\
\hline Curtosis & -1.641 & -1.250 \\
\hline Mínimo & 3 & 1 \\
\hline Máximo & 6 & 4 \\
\hline
\end{tabular}

Las visitas representan los accesos que se hacen a la plataforma virtual con el fin de ingresar al aula y ver las actividades de cada alumno. Es posible observar que cada una de las actividades tanto colaborativas como en las evidencias de aprendizaje, se tuvo una revisión promedio de 4.7 visitas por parte del estudiante, las cuales se traducen en actividad de revisión, lectura e intercambio de información; de este modo los alumnos con menos afluencia en el envío de tareas, tuvieron un índice mínimo de 3 visitas mientras que el máximo de visitas por tarea fue de 6 ocasiones.

Para el caso de las retroalimentaciones hechas por el tutor a cada una de las actividades realizadas por los alumnos se reporta un promedio de 3.07, lo cual implica el intercambio de información entre alumno-tutor a fin de hacer modificación de actividades, ampliar información, atender comentarios o aclarar dudas relativas al contenido o a la forma de la actividad. Se posibilitó el desarrollo de al menos una acción de retroalimentación o seguimiento para cada alumno, llegando al nivel de 4 retroalimentaciones por tarea de cada alumno.

En el caso de las retroalimentaciones por alumno se tuvo la intención de generar un programa en el cual cada alumno recibiera indicaciones particulares sobre su desempeño; en este sentido, había comunicación por mensajería personal en función de la actividad que cada uno de ellos presentó, habiendo una comunicación de 16.15 mensajes individuales en promedio por cada persona, con una desviación de 2.98 respecto a la media, lo cual implica un nivel de dispersión que hace ver que cada alumno tuvo una frecuencia de envío muy similar. El alumno que menos mensajes 
recibió (8 mensajes) fue en virtud a la poca o nula respuesta que hubo de parte de ellos, mientras que el alumno que más mensajes recibió (20 mensajes), fue a partir de que ellos mismos incluso incrementaron su demanda de comunicación a partir de la actividad que realizaron.

Un elemento que parece fundamental estudiar, corresponde a revisar si el factor de tiempo en la retroalimentación que recibe el alumno resulta importante para el alumno en su actividad dentro del módulo. Para corroborarlo, se hace un análisis sobre la correlación que existió entre el tiempo que hubo en la entrega de una tarea, participación o calificación, respecto a recibir una retroalimentación o calificación, a lo que en este estudio se le ha llamado latencia basada en días, a lo cual tenemos el siguiente resultado:

Tabla 5 .

Tiempo promedio de retroalimentación

\begin{tabular}{|c|c|}
\hline Media & 3.9722 \\
Desv. típ. & 1.78715 \\
Mínimo & 1.00 \\
Máximo & 8.00 \\
\hline
\end{tabular}

El conteo se hace en función a días tomando en cuenta que estos se fraccionaron en segmentos basados en horas; de esta forma disponemos de un tiempo promedio de 3.97 días en que el alumno envía una actividad y recibía una retroalimentación; con una desviación de 1.78, la cual se vuelve con una escasa dispersión respecto a dicha media y en donde el tiempo mínimo en el que el alumno recibió una retroalimentación fue de 1 día, esperando como máximo un periodo de 8 días para ello.

En función a que el tiempo equivalió a tener comunicación en un sentido de motivación y de cobertura formativa, resultó crucial su análisis para saber su efecto sobre el resultado obtenido por cada alumno. De este modo, se aplica un análisis de comparación de medias para muestras independientes a fin de distinguir este efecto, haciendo una diferencia sobre aquellos alumnos que aprobaron, respecto a quienes no lo hicieron o no presentaron actividad; teniendo una $\mathrm{T}(25)=-4.25, \mathrm{P}=$ o.oo lo cual nos indica que si existió una diferencia significativa entre los alumnos que aprobaron y los que no aprobaron, respecto al tiempo en el que se les fue retroalimentando. 


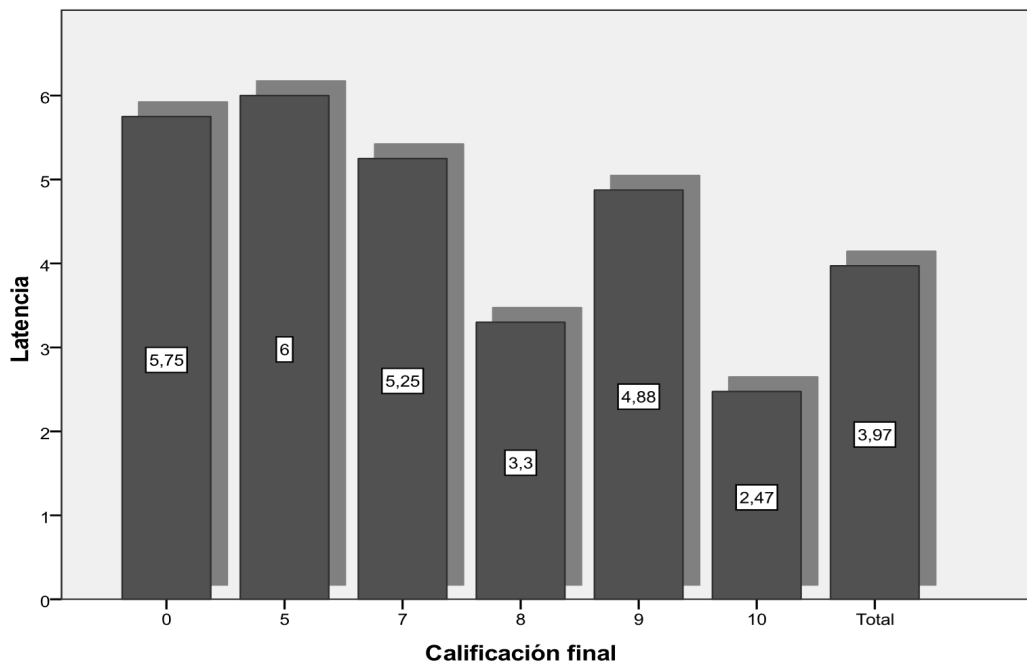

Figura 4. Tiempo promedio de latencia en días, en retroalimentación y la calificación final

La figura 4 nos muestra el periodo promedio de latencia en días respecto a la calificación final, lo cual permite corroborar que existe diferencia en aquellos alumnos que abandonan el módulo (calificación o) y los que no aprobaron (calificación 5), respecto a quienes lograron acreditar. Incluso es posible observar que aunque la latencia no fue inversamente proporcional con el resultado final obtenido por el alumno, aun así fue posible considerar un efecto de la variable del tiempo con relación a la calificación final.

En cuanto a saber si la calificación final tuvo algún efecto significativo respecto a cada acción individual en la dinámica del tutor, se hizo un análisis de varianza considerando también la diferencia entre aquellos alumnos que aprobaron, con respecto a quienes no lo hicieron, encontrando los resultados siguientes:

Tabla 6.

Análisis de varianza de la frecuencia de actividad del tutor

\begin{tabular}{|l|l|}
\hline \multicolumn{1}{|c|}{ Variable de actividad docente } & \multicolumn{1}{c|}{ Correlación } \\
\hline Frecuencia de visitas tarea/alumno & $\mathrm{T}(25)=1.424, p=0.167$ \\
\hline Frecuencia de retroalimentación tarea/alumno & $\mathrm{T}(25)=4.697, p=0.000$ \\
\hline Frecuencia de mensajes enviados por alumno & $\mathrm{T}(25)=-1.181, p=0.249$ \\
\hline
\end{tabular}


El análisis entre las distintas acciones ejercidas por el tutor respecto al resultado obtenido en función a si aprobaron o no, resultó que no todos mostraron un efecto significativamente distinto, habiendo solo una relación significativa en cuanto a la frecuencia de retroalimentaciones que se brinda a los alumnos, mientras que las visitas que haga el tutor y la cantidad de mensajes que envía a cada alumno parecen no mostrar efectos importantes respecto al desempeño final del alumno.

- Efectos de la acción tutorial sobre el desempeño y habilidades de los alumnos

Una parte importante de este análisis corresponde a determinar el efecto que existe de las variables que describen la participación o acción del docente, sobre los resultados y desempeño de los alumnos.

Se aplicó un análisis de varianza factorial a los datos de las acciones de la acción del tutor comparando su efecto sobre el resultado final del grupo, donde se registraron algunas diferencias significativas respecto de las formas específicas de acción del tutor y el resultado final (tabla 7).

\section{Tabla 7.}

Resultado del análisis de varianza para las diversas acciones del tutor sobre el resultado del grupo

\begin{tabular}{|cc|c|c|c|c|c|}
\hline & & $\begin{array}{c}\text { Suma de } \\
\text { cuadrados }\end{array}$ & gl & $\begin{array}{c}\text { Media } \\
\text { cuadrática }\end{array}$ & F & Sig. \\
\hline Número de visitas por & Inter-grupos & 3.163 & 2 & 1.581 & 1.041 & .369 \\
tarea/individuo & $\begin{array}{c}\text { Intra-grupos } \\
\text { Total }\end{array}$ & 36.467 & 24 & 1.519 & & \\
\hline Número de & Inter-grupos & 14.185 & 2 & 7.093 & 10.865 & .000 \\
retroalimentaciones por & Intra-grupos & 15.667 & 24 & .653 & & \\
tarea/individuo & Total & 29.852 & 26 & & & \\
\hline Tiempo promedio de & Inter-grupos & 34.967 & 2 & 17.483 & 8.728 & .001 \\
retroalimentación & Intra-grupos & 48.075 & 24 & 2.003 & & \\
\hline \multirow{2}{*}{ Mensajes individuales } & Total & 83.042 & 26 & & & \\
\hline enviados & Inter-grupos & 14.907 & 2 & 7.454 & .826 & .450 \\
\hline \multirow{2}{*}{ Intra-grupos } & 216.500 & 24 & 9.021 & & \\
\hline \multirow{2}{*}{ Talidad en latencia } & Inter-grupos & 12.050 & 2 & 6.025 & 7.014 & .004 \\
& Intra-grupos & 20.617 & 24 & .859 & & \\
\hline & Total & 32.667 & 26 & & & \\
\hline
\end{tabular}


Es posible observar que no existen diferencias entre las dos formas de acción relacionadas por un lado con el número de visitas que el tutor hace a las tareas de cada individuo $(\mathrm{F}(2,24)=1.041, \mathrm{p}=.369)$ y la que corresponde al número de mensajes individuales que el tutor hace a cada individuo $(\mathrm{F}(2,24)=0.826, \mathrm{p}=$ .450); determinando en ambos casos que existe una acción directa de parte del tutor sobre el alumno en forma individual y que esto no representó un cambio sobre el resultado de los alumnos.

Para el caso de los elementos relacionados con el número de retroalimentaciones, el tiempo promedio que se tarda en dar una retroalimentación a partir de haberse presentado una tarea y el resultado de ese tiempo o latencia sobre la acción de los alumnos; parece ser determinante en el sentido de mostrar diferencias significativas importantes. Scheffé.

Con el fin de identificar el sentido de las diferencias, se aplicó la prueba de

Tabla 8.

Comparaciones múltiples de los resultados obtenidos con los indicadores de actividad docente

\begin{tabular}{|c|c|c|c|c|c|c|c|}
\hline \multirow[t]{2}{*}{$\begin{array}{c}\text { Variable } \\
\text { dependiente }\end{array}$} & \multirow{2}{*}{$\begin{array}{l}\text { (l) } \\
\text { Resultado } \\
\text { final en } \\
\text { módulo }\end{array}$} & \multirow{2}{*}{$\begin{array}{l}\text { (J) Resultado } \\
\text { final en } \\
\text { módulo }\end{array}$} & \multirow{2}{*}{$\begin{array}{l}\text { Diferencia } \\
\text { de medias } \\
\quad \text { (I-J) }\end{array}$} & \multirow{2}{*}{$\begin{array}{l}\text { Error } \\
\text { típico }\end{array}$} & \multirow[t]{2}{*}{ Sig. } & \multicolumn{2}{|c|}{$\begin{array}{l}\text { Intervalo de } \\
\text { confianza } 95 \%\end{array}$} \\
\hline & & & & & & $\begin{array}{l}\text { Límite } \\
\text { inferior }\end{array}$ & $\begin{array}{l}\text { Límite } \\
\text { superior }\end{array}$ \\
\hline \multirow{6}{*}{$\begin{array}{l}\text { Número de } \\
\text { visitas por tarea/ } \\
\text { individuo }\end{array}$} & \multirow{2}{*}{ Ausente } & No aprobado & .333 & .941 & .939 & -2.12 & 2.79 \\
\hline & & Aprobado & -.567 & .763 & .761 & -2.56 & 1.42 \\
\hline & \multirow{2}{*}{$\begin{array}{c}\text { No } \\
\text { aprobado }\end{array}$} & Ausente & -.333 & .941 & .939 & -2.79 & 2.12 \\
\hline & & Aprobado & -.900 & .675 & .424 & -2.66 & .86 \\
\hline & \multirow{2}{*}{ Aprobado } & Ausente & .567 & .763 & .761 & -1.42 & 2.56 \\
\hline & & No aprobado & .900 & .675 & .424 & -.86 & 2.66 \\
\hline \multirow{6}{*}{$\begin{array}{l}\text { Número de } \\
\text { retroalimenta- } \\
\text { ciones por tarea/ } \\
\text { individuo }\end{array}$} & \multirow{2}{*}{ Ausente } & No aprobado & -.333 & .617 & .865 & -1.94 & 1.28 \\
\hline & & Aprobado & $-1.833^{*}$ & .500 & .005 & -3.14 & -.53 \\
\hline & \multirow{2}{*}{$\begin{array}{c}\text { No } \\
\text { aprobado }\end{array}$} & Ausente & .333 & .617 & .865 & -1.28 & 1.94 \\
\hline & & Aprobado & $-1.500^{*}$ & .443 & .009 & -2.65 & -.35 \\
\hline & \multirow{2}{*}{ Aprobado } & Ausente & $1.833^{*}$ & .500 & .005 & .53 & 3.14 \\
\hline & & No aprobado & $1.500^{*}$ & .443 & .009 & .35 & 2.65 \\
\hline
\end{tabular}




\begin{tabular}{|c|c|c|c|c|c|c|c|}
\hline \multirow[t]{2}{*}{$\begin{array}{c}\text { Variable } \\
\text { dependiente }\end{array}$} & \multirow{2}{*}{$\begin{array}{l}\text { (l) } \\
\text { Resultado } \\
\text { final en } \\
\text { módulo }\end{array}$} & \multirow{2}{*}{$\begin{array}{l}\text { (J) Resultado } \\
\text { final en } \\
\text { módulo }\end{array}$} & \multirow{2}{*}{$\begin{array}{c}\text { Diferencia } \\
\text { de medias } \\
\text { (I-J) }\end{array}$} & \multirow{2}{*}{$\begin{array}{l}\text { Error } \\
\text { típico }\end{array}$} & \multirow[t]{2}{*}{ Sig. } & \multicolumn{2}{|c|}{$\begin{array}{c}\text { Intervalo de } \\
\text { confianza 95\% }\end{array}$} \\
\hline & & & & & & $\begin{array}{l}\text { Límite } \\
\text { inferior }\end{array}$ & $\begin{array}{l}\text { Límite } \\
\text { superior }\end{array}$ \\
\hline \multirow{6}{*}{$\begin{array}{c}\text { Tiempo } \\
\text { promedio de } \\
\text { retroalimentación }\end{array}$} & \multirow{2}{*}{ Ausente } & No aprobado & -.25000 & 1.08097 & .974 & -3.0700 & 2.5700 \\
\hline & & Aprobado & $2.45000^{*}$ & .87628 & .034 & .1640 & 4.7360 \\
\hline & \multirow{2}{*}{$\begin{array}{c}\text { No } \\
\text { aprobado }\end{array}$} & Ausente & .25000 & 1.08097 & .974 & -2.5700 & 3.0700 \\
\hline & & Aprobado & $2.70000^{*}$ & .77520 & .007 & .6777 & 4.7223 \\
\hline & \multirow{2}{*}{ Aprobado } & Ausente & $-2.45000^{*}$ & .87628 & .034 & -4.7360 & -.1640 \\
\hline & & No aprobado & $-2.70000^{*}$ & .77520 & .007 & -4.7223 & -.6777 \\
\hline \multirow{6}{*}{$\begin{array}{l}\text { Mensajes } \\
\text { individuales } \\
\text { enviados }\end{array}$} & \multirow{2}{*}{ Ausente } & No aprobado & & 2.294 & .863 & -4.73 & 7.23 \\
\hline & & Aprobado & 2.250 & 1.860 & .491 & -2.60 & 7.10 \\
\hline & \multirow{2}{*}{$\begin{array}{c}\text { No } \\
\text { aprobado }\end{array}$} & & -1.250 & 2.294 & .863 & -7.23 & 4.73 \\
\hline & & Aprobado & 1.000 & 1.645 & .832 & -3.29 & 5.29 \\
\hline & \multirow{2}{*}{ Aprobado } & Ausente & -2.250 & 1.860 & .491 & -7.10 & 2.60 \\
\hline & & No aprobado & -1.000 & 1.645 & .832 & -5.29 & 3.29 \\
\hline \multirow{6}{*}{$\begin{array}{l}\text { Calidad en } \\
\text { latencia }\end{array}$} & \multirow{2}{*}{ Ausente } & No aprobado & -.167 & .708 & .973 & -2.01 & 1.68 \\
\hline & & Aprobado & $-1.617^{*}$ & .574 & .032 & -3.11 & -.12 \\
\hline & \multirow{2}{*}{$\begin{array}{c}\text { No } \\
\text { aprobado }\end{array}$} & Ausente & .167 & .708 & .973 & -1.68 & 2.01 \\
\hline & & Aprobado & $-1.450^{*}$ & .508 & .030 & -2.77 & -.13 \\
\hline & \multirow{2}{*}{ Aprobado } & Ausente & $1.617^{*}$ & .574 & .032 & .12 & 3.11 \\
\hline & & No aprobado & $1.45 \mathrm{O}^{*}$ & .508 & .030 & .13 & 2.77 \\
\hline
\end{tabular}

*. La diferencia de medias es significativa al nivel .05.

Es posible observar algunas diferencias relacionadas específicamente con el grupo de los alumnos que aprobaron, a diferencia de quienes no lo hicieron o se ausentaron. Estas diferencias son principalmente las siguientes:

Por una parte los alumnos aprobados difieren de los demás a partir del número de retroalimentaciones que da el tutor a cada alumno, haciendo evidente que a mayor número de retroalimentaciones individuales, la posibilidad de aprobación, generación de habilidades de autorregulación, motivación y asimilación de contenidos aumenta. Por otra parte, el mismo efecto parece determinar el tiempo promedio en el cual el tutor retroalimenta, en el hecho de que parece existir una relación en la cual a menor tiempo promedio en el cual un alumno presenta sus trabajos del módulo y el tutor le retroalimenta y califica, pareciera que esto aumenta considerablemente las posibilidades de aprobación de los alumnos.

Con el fin de determinar el efecto de la latencia sobre el resultado de los alumnos y sus dinámicas de participación y cambio, se hace el análisis de la calidad en Latencia, el cual representa el resultado que existe entre el tiempo de retroalimentación y la calificación obtenida; con ello queda demostrado que si se 
realiza una retroalimentación en tiempo breve, el alumno dispone de la posibilidad de generar mejoras, de motivarse para hacer búsquedas y precisiones, así como de obtener mejores resultados y logros. Para este caso, se presentó una diferencia significativa en los alumnos que aprobaron con respecto a quienes no lo hicieron.

\section{CONCLUSIONES}

La autorregulación parece tener una relación directa con el diseño instruccional y la dinámica de comunicación de los tutores docentes. Tomemos en cuenta que las personas que ingresan a sistemas de educación a distancia no tienen un amplio dominio en el uso de tecnología de la educación, y que en su caso más próximo se relacionan con tecnologías comerciales en donde las aplicaciones en dispositivos móviles, smartphones, tablets o i-pad son cotidianamente utilizadas para el desarrollo de actividades recreativas o de trasmisión de información; pero no así para potenciar su aplicación a desarrollos tecnológicos, lo cual trae consigo problemas que se ven reflejados en el avance de su carrera. Pues bien, esto tiende a incrementar a partir del hecho de que las personas que fungen como tutores cuentan con carencias similares.

Entre los datos que se han encontrado en el presente estudio, se puede deducir que el diseño que los tutores realizan respecto a la forma en la que programan, controlan, distribuyen, retroalimentan y evalúan; no solo propicia afectación en los resultados de rendimiento de los alumnos, sino que además pudiera posibilitar el incremento en el aprendizaje de habilidades de autorregulación de sus alumnos. El papel del tutor dentro de un sistema a distancia, implica necesariamente el conocimiento del contenido temático que pretende incluir en la formación de los alumnos, pero además implica un perfil que incluya el conocimiento de las principales herramientas tecnológicas, su uso común y clasificación. En el presente estudio se utilizaron herramientas disponibles para la versión de Moodle que se usó en el CUVED, así como un diseño instruccional que pudiera permitir su uso común. Para el caso, se pudo observar que los alumnos recurren a herramientas que les permitan el logro de al menos tres aspectos: Obtención de la información temática necesaria (más no única) para el desarrollo de las unidades, objetos que les brinden identidad tanto institucional como personal y, principalmente, recursos que les permitan dimensionar la carga de actividad formativa con respecto al tiempo disponible.

De esto es posible prescribir que para facilitar la acción tutora en ambientes virtuales con el desarrollo de habilidades de autorregulación en los alumnos, debemos al menos procurar por brindarles todos los materiales y recursos mínimos necesarios para el cumplimiento de los objetivos y no basarnos en el posible precepto de que los alumnos son "expertos buscadores" de información usando solamente navegadores. Debemos procurar por el desarrollo de objetos que les permitan crear una personalización de sus espacios (Moodle incluso permite el uso de estos perfiles) 
y una identificación directa con la institución; esto puede ser mediante objetos que puedan basarse desde la multimedia y hasta el uso de información que les propicie identidad en redes sociales o en sistemas de geolocalización o de inclusión a actividades extracurriculares en su misma institución. Un punto adicional y quizá el más importante, es el que corresponde a crear elementos que permita a los alumnos poder dimensionar todas la actividades por realizar y el tiempo disponible para su desarrollo; el logro de estos recursos puede estar basado en el establecimiento de cronogramas y hasta el uso sincronizado de agendas virtuales vinculadas con cuentas de google, como puede ser un ejemplo.

Es preciso señalar entonces que en un proceso de educación a distancia a través del uso de tecnologías, se requiere que el perfil del tutor adquiera habilidades y estrategias encausadas a tres condiciones: 1) Que propicie todos los materiales y recursos para el desarrollo óptimo de su ejercicio formativo, apelando a la existencia de habilidades de búsqueda por parte de los alumnos, siempre y cuando sea esta habilidad adquirida y supervisada por el mismo tutor, y no partir de la noción de que el alumno ya sabe realizar búsquedas especializadas y distinguir entre los recursos con buen nivel y los que no lo son. 2) Que genere posibilidades de personalización de espacios en sus perfiles y brinde recursos que propicien el apego e identificación con la institución y con sus propias metas. 3) Que brinde información en donde se dosifique toda la actividad formativa y los tiempos establecidos para la misma desde el principio, asumiendo que hay tanto alumnos de tiempo completo, como alumnos que cuentan con otras prioridades; pero que ambos grupos requieren saber qué es lo que deben hacer y en qué tiempo hay que hacerlo.

Por otra parte, es necesario señalar que la intención de este trabajo no ha sido propiamente el proponer un espacio virtual específico como único ejemplo de desarrollo de habilidades autorregulatorias en educación a distancia; por el contrario, actualmente existen muchos sistemas tecnológicos que permiten este y muchos otros elementos que brindan la posibilidad de desarrollo. Sin embargo, tampoco podemos dejar de lado que el uso de ciertos entornos virtuales propician de mejor manera el desarrollo de la educación a distancia y, particularmente, la adecuación que se hizo en el CUVED permitió en forma óptima que se generaran las habilidades en los alumnos.

Cuando se diseñó este espacio, se procuró por partir del hecho de que la tecnología no es el punto central del desarrollo de un sistema de educación; la idea es considerar el antecedente histórico de depositar el éxito de los sistemas e-learning y b-learning en la funcionalidad de sus sistemas virtuales, siendo que estos tienden a devaluarse o a interrumpirse aceleradamente. La construcción del CUVED estuvo supeditada a los preceptos básicos de los modelos de aprendizaje autorregulado y de los mecanismos válidos de evaluación e instrucción. 
En este sentido se adecuó Moodle a fin de que se cubran los objetivos señalados y mantener una postura que propiciara la interacción. Dicha postura consiste en: mantener la misma línea de enseñanza en los módulos que se han incluido en CUVED con el fin de procurar resultados equivalentes. Partiendo del Teorema de Equivalencia que maneja Garrison y Anderson (2003), es posible considerar el efecto de la interacción entre contenidos, alumnos y tutores.

Dicho teorema describe un conjunto de interacciones utilizando la versatilidad de los recursos tecnológicos, los cuales propician la migración de interacciones. Dicho teorema señala:

- Se pueden desarrollar niveles profundos de aprendizaje significativo siempre que una de las tres partes principales de formas de interacción (alumnoprofesor, alumno-alumno o alumno-contenido) se dé en un nivel muy elevado. Las otra dos se pueden dar en niveles mínimos o incluso podrían ser eliminadas del todo, sin que ello conlleve a un deterioro de la acción educativa.

- Cuando el nivel de interacción es elevado en más de una forma de estas tres, resulta mayor el grado de satisfacción que se experimenta con la acción educativa (Anderson, 2003).

La base principal del desarrollo de estas interacciones se encuentra en el diseño instruccional que el tutor disponga en el momento de construir el espacio de aprendizaje.

Se consideró por otra parte, que existen tres tipos de evidencias que analizan la eficacia de la instrucción: a) la acción individual del tutor, b) el aprendizaje colaborativo, y c) los recursos multimedia. Aunque evidentemente los recursos pueden generar resultados diferentes de interacción, nos permite tener diferentes canales de información respecto al desarrollo de habilidades de aprendizaje autorregulado en los alumnos.

A partir de estas condiciones, fue posible construir el espacio virtual de CUVED y adecuar el contenido del módulo. CUVED permitió incluir elementos basados en actividades colaborativas, actividades de participación individual y evidencias de aprendizaje basadas en los contenidos. A su vez, fue posible incluir elementos de evaluación con indicadores de validez y confiabilidad necesarias para generar una adecuación más pertinente.

Dentro de este estudio, fue posible reconocer la importancia y pertinencia de que las actividades de trabajo tengan un marco de equilibrio en su desarrollo, sin embargo, fue posible determinar que mientras exista un diseño que tenga por 
intención el desarrollo de alguna de las interacciones, es posible que se desarrolle con éxito la actividad formativa. Para el caso de SUAED Psicología, fue posible determinar que sus alumnos tienden más hacia el modo de interacción entre ellos mismos con los recursos, desde una dinámica individual y con una evaluación también individual, mientras que la labor de la acción docente radica principalmente en la idea de dosificar recursos y actividades, con una comunicación constante, motivación basada en metas e identidad universitaria, así como en el seguimiento de su formación a través del fomento de las demás interacciones. Vale bien la pena poder averiguar sobre la pertinencia en la cantidad y calidad de las actividades y materiales que sean óptimos para el desarrollo de módulos y materias de formación en un sistema de educación a distancia.

\section{REFERENCIAS BIBLIOGRÁFICAS}

Anderson, T. (2003). Modes of Interacción in Distance Education: Recent Developments and Research Questions. En M. Moore y G. Anderson (eds.). Handbook of Distance Education, (pp. 129-145). Mahwah, NJ, Erlbaum.

Anderson, T., y Kuskis. A. (2007). Modes of interaction. En M. Grahame, Handbook of Distance Education (pp. 295 309). New Jersey: Lawrence Erlbaum Associates, Publishers.

Anderson, T., Rourke, L., Garrison, D. R., y Archer, W. (2001). Assessing teaching presence in a computer conferencing context. Journal of Asynchronous Learning Networks, 5(2).

Azevedo, R. (2005). Using hypermedia as a metacognitive tool for enhancing student learning? The role of the self regulated learning. Educational Psychologist, 40, 199-209.

Baker, J. D. (2004). An investigation of relationship among instructor immediacy and effective and cognitive learning in the online classroom. The Internet and Higher Education, 7(1), 1-13.

Garrison, D. R., y Anderson, T. (2003). E-learning in the 21st century: $A$ framework for research and practice. London: Routledge/Falmer.
Katz, Y. (2000). The comparative suitability of three ICT distance learning methodologies for college level instruction. Education Media International, 37(1), 25-30.

McIsaac, M. S., y Gunawardena, C. N. (1996). Distance Education. En H. D. Jonassen, (Ed.). Handbook of Reseach for Educational Communications and Technology: A projest of the association for educational communications and technology, (pp. 403-437). New York: Simon \& Schuster.

Simonson, M., Sharon, S., Albright, M., y Zvacek, S. (2003). Teaching and Learning at a Distance. USA: Merrill Prentice Hall.

Vohs, K., y Ciarocco, N. (2004). Interpersonal functioning requires self-regulation. En R. Baumesiter, y K. Vohs. Handbook of Self-Regulation: research, theory and applications. (pp. 392-407) New York: The Guilford Press.

Wang, Y. (2004). Supporting synchronous distance language learning with desktop videoconferencing. Language Learning \& Technology, 8(3), 1-37. 


\section{PERFIL ACADÉMICO Y PROFESIONAL DEL AUTOR}

Omar Moreno Almazán. Psicólogo por la UNAM desde 2001 y Doctorado en Psicología Educativa y del Desarrollo. Docente en sistemas de educación e-learning con certificación nacional; ha desarrollado cursos, talleres, diplomados, así como participado en la elaboración de materiales digitales, multimedia en adecuaciones curriculares en sistemas de educación a distancia. Ha sido jefe en la misma área. Investiga sobre procesos de evaluación, planificación e intervención educativa basada en tecnología.

E-mail: almazanomar@gmail.com

DIRECCIÓN DEL AUTOR

UNAM FES Iztacala

Coordinación de Educación a Distancia Av. De los Barrios No. 1, Los Reyes Iztacala; Tlalnepantla, Estado de México. México.

Fechas de recepción del artículo: 18/05/2014

Fecha de aceptación del artículo: 24/07/2014

\section{Como citar este artículo:}

Moreno Almazán, O. (2015). Evaluación de la modalidad de interacción de la tutoría y los efectos en logro académico en entornos en línea. RIED. Revista Iberoamericana de educación a Distancia, volumen 18, $\mathrm{n}^{0}$ 1, pp. 231-255. 